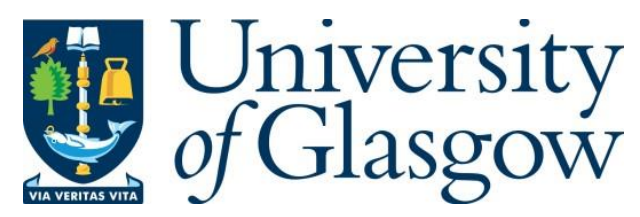

Bartel, A. and Page, A. (2016) Torsion homology and regulators of isospectral manifolds. Journal of Topology, 9(4), pp. 1237-1256.

There may be differences between this version and the published version. You are advised to consult the publisher's version if you wish to cite from it.

$\underline{\text { http://eprints.gla.ac.uk/146951/ }}$

Deposited on: 11 October 2017

Enlighten - Research publications by members of the University of Glasgow http://eprints.gla.ac.uk 


\title{
TORSION HOMOLOGY AND REGULATORS OF ISOSPECTRAL MANIFOLDS
}

\author{
ALEX BARTEL AND AUREL PAGE
}

\begin{abstract}
Given a finite group $G$, a $G$-covering of closed Riemannian manifolds, and a so-called $G$-relation, a construction of Sunada produces a pair of manifolds $M_{1}$ and $M_{2}$ that are strongly isospectral. Such manifolds have the same dimension and the same volume, and their rational homology groups are isomorphic. Here, we investigate the relationship between their integral homology. The Cheeger-Müller Theorem implies that a certain product of orders of torsion homology and of regulators for $M_{1}$ agrees with that for $M_{2}$. We exhibit a connection between the torsion in the integral homology of $M_{1}$ and $M_{2}$ on the one hand, and the $G$-module structure of integral homology of the covering manifold on the other, by interpreting the quotients $\operatorname{Reg}_{i}\left(M_{1}\right) / \operatorname{Reg}_{i}\left(M_{2}\right)$ representation theoretically. Further, we prove that the $p^{\infty}$-torsion in the homology of $M_{1}$ is isomorphic to that of $M_{2}$ for all primes $p \nmid \# G$. For $p \leq 71$, we give examples of pairs of strongly isospectral hyperbolic 3 -manifolds for which the $p$-torsion homology differs, and we conjecture such examples to exist for all primes $p$.
\end{abstract}

\section{INTRODUCTION}

Two closed Riemannian manifolds $M_{1}$ and $M_{2}$ are said to be isospectral if the Laplace-Beltrami operators acting on functions on $M_{1}$ and on $M_{2}$ have the same spectrum, equivalently if the spectral zeta functions $\zeta\left(M_{1}, s\right)$ and $\zeta\left(M_{2}, s\right)$ are equal. The manifolds are said to be strongly isospectral if the spectra of every natural self-adjoint elliptic differential operator on $M_{1}$ and $M_{2}$ agree. In particular, if $M_{1}$ and $M_{2}$ are strongly isospectral, then the Laplace-de Rham operators acting on differential $i$-forms on the respective manifold have the same spectrum, equivalently the zeta functions $\zeta_{i}\left(M_{1}, s\right)$ and $\zeta_{i}\left(M_{2}, s\right)$ encoding those spectra are equal. Following Kac's famous question "Can one hear the shape of a drum?" [19, the following broad questions have received a lot of attention [14, 15.

Question 1.1. Which isometry invariants of closed Riemannian manifolds are isospectral invariants? Which ones are strongly isospectral invariants?

For example, two Riemannian manifolds that are strongly isospectral have the same dimension, volume and Betti numbers.

By analogy with the number theoretic notion of so-called arithmetically equivalent number fields, Sunada [35] proposed a general construction showing that there exist Riemannian manifolds that are strongly isospectral,

Mathematics Institute, Zeeman Building, University of Warwick, Coventry CV4 7AL, UK

E-mail addresses: a.bartel@warwick.ac.uk, a.r.page@warwick.ac.uk.

The authors thank the EPSRC for financial support via a First Grant, respectively the EPSRC Programme Grant EP/K034383/1 LMF: L-Functions and Modular Forms. 
but are not even homeomorphic. Let us briefly recall Sunada's construction. Let $X \rightarrow Y$ be a $G$-covering of closed manifolds, where $G$ is a finite group. Let $U_{1}, U_{2}$ be two subgroups of $G$ with the property that there is an isomorphism $\mathbb{C}\left[G / U_{1}\right] \cong \mathbb{C}\left[G / U_{2}\right]$ of linear permutation representations of $G$, or equivalently that for every conjugacy class $c$ of $G$ we have $\#\left(c \cap U_{1}\right)=\#\left(c \cap U_{2}\right)$. In this case we say that the formal linear combination $U_{1}-U_{2}$ is a $G$-relation. Sunada proves that then the intermediate coverings $X / U_{1}$ and $X / U_{2}$ are strongly isospectral.

In this paper, we want to take the analogy with number fields further. The Cheeger-Müller Theorem [11, 22, 23], proving a conjecture of Ray and Singer [30], gives a special value formula for the spectral zeta functions of a Riemannian manifold, somewhat analogous to the analytic class number formula for the Dedekind zeta function of a number field [36]. It implies that if $M_{1}$ and $M_{2}$ are strongly isospectral Riemannian manifolds, then

$$
\prod_{i=0}^{d}\left(\frac{\operatorname{Reg}_{i}\left(M_{1}\right)}{\# H_{i}\left(M_{1}, \mathbb{Z}\right)_{\text {tors }}}\right)^{(-1)^{i}}=\prod_{i=0}^{d}\left(\frac{\operatorname{Reg}_{i}\left(M_{2}\right)}{\# H_{i}\left(M_{2}, \mathbb{Z}\right)_{\text {tors }}}\right)^{(-1)^{i}}
$$

where, for a Riemannian manifold $M, \operatorname{Reg}_{i}(M)$ is the covolume of the lattice $H_{i}(M, \mathbb{Z}) / H_{i}(M, \mathbb{Z})_{\text {tors }}$ in the vector space $H_{i}(M, \mathbb{R})$ with respect to a certain canonical inner product (see Notation 3.2 ii.). The study of torsion homology and regulators of manifolds has attracted a lot of attention recently, see for instance [4, 5, 8, 10].

In light of equation (1.2), a natural instance of Question 1.1 is whether, for $M_{1}$ and $M_{2}$ as above, an integer $i \geqslant 0$, and a prime number $p$, we must necessarily have

$$
\# H_{i}\left(M_{1}, \mathbb{Z}\right)\left[p^{\infty}\right]=\# H_{i}\left(M_{2}, \mathbb{Z}\right)\left[p^{\infty}\right]
$$

where $\left[p^{\infty}\right]$ denotes the $p$-primary torsion subgroup.

Here, we answer this and similar questions in the context of Sunada's construction. More broadly, we study homological properties of covering manifolds in $G$-relations, analogous to an old and fruitful line of research in number theory. For example, the following basic result is analogous to results of [6] on class groups of number fields.

Proposition 1.4. Let $X \rightarrow Y$ be a G-covering of closed Riemannian manifolds, let $U_{1}-U_{2}$ be a $G$-relation, and let $i \geqslant 0$ be an integer. Then for all primes $p$ that do not divide $\# G$, we have

$$
H_{i}\left(X / U_{1}, \mathbb{Z}\right)\left[p^{\infty}\right] \cong H_{i}\left(X / U_{2}, \mathbb{Z}\right)\left[p^{\infty}\right] .
$$

We actually prove a more general result, see Theorem 3.5 .

In providing counter examples, we have concentrated on closed hyperbolic 3-manifolds, a class of manifolds that plays an important rôle in other branches of geometry, as well as in number theory. The following is a result in the opposite direction to that of Proposition 1.4.

Proposition 1.5. Let $p \leq 71$ be a prime number. Then there exist strongly isospectral closed hyperbolic 3-manifolds $M_{1}$ and $M_{2}$ such that

$$
\# H_{1}\left(M_{1}, \mathbb{Z}\right)\left[p^{\infty}\right] \neq \# H_{1}\left(M_{2}, \mathbb{Z}\right)\left[p^{\infty}\right] .
$$


Moreover, if $2<p \leq 71$, then there exist 3 -manifolds $M_{1}$ and $M_{2}$ as above with

$$
\# H_{1}\left(M_{1}, \mathbb{Z}\right)\left[p^{\infty}\right]=1, \quad \text { and } \quad \# H_{1}\left(M_{2}, \mathbb{Z}\right)\left[p^{\infty}\right]=p .
$$

Note that hyperbolic 3-manifolds have torsion-free homology in degrees 0 , 2 , and 3 , so in the situation of Proposition 1.5 , equation $(1.3)$ is trivially satisfied in those degrees.

It is already known that for every prime $p$, there exist strongly isospectral closed Riemannian 4-manifolds $M_{1}$ and $M_{2}$ such that $\# H_{1}\left(M_{1}, \mathbb{Z}\right)\left[p^{\infty}\right] \neq$ $\# H_{1}\left(M_{2}, \mathbb{Z}\right)\left[p^{\infty}\right]$. This is shown in [35], using the following two results. Firstly, every finite group can be realised as the fundamental group of a closed smooth 4-manifold [33, p. 402]. Secondly, for every prime $p$, there exists a finite $p$-group $G$ with a $G$-relation $U_{1}-U_{2}$, where the abelianisations of $U_{1}$ and $U_{2}$ have different orders [35, §1, Example 3].

At the other extreme, if $M$ is a closed oriented 2-manifold, then $H_{1}(M, \mathbb{Z})$ is torsion-free. Riemannian 3-manifolds present an in-between case. It follows from the Elliptisation Theorem, as proven by Perelman [26, 27, 28, and from a theorem of Ikeda [18, that any two isospectral 3-manifolds with finite fundamental groups are homeomorphic. In particular, Sunada's construction for 4-manifolds cannot possibly generalise to 3-manifolds. Instead, we prove Proposition 1.5 by a judicious choice of $G$-relation $U_{1}-U_{2}$, a different one for each prime $p$, and then by performing a computer search among $G$-coverings of hyperbolic 3-manifolds and computing the resulting torsion homology of the intermediate coverings corresponding to $U_{1}$ and $U_{2}$. Nevertheless, we believe that the evidence of Proposition 1.5.justifies the following conjecture ${ }^{1}$

Conjecture 1.6. For every prime number $p$, there exist strongly isospectral closed hyperbolic 3 -manifolds $M_{1}$ and $M_{2}$ such that $\# H_{1}\left(M_{1}, \mathbb{Z}\right)\left[p^{\infty}\right] \neq$ $\# H_{1}\left(M_{2}, \mathbb{Z}\right)\left[p^{\infty}\right]$.

It follows from equation 1.2 that if $M_{1}$ and $M_{2}$ are strongly isospectral, then $\prod_{i}\left(\operatorname{Reg}_{i}\left(M_{1}\right) / \operatorname{Reg}_{i}\left(M_{2}\right)\right)^{(-1)^{i}}$ can be expressed in terms of torsion homology of the two manifolds, and in particular is a rational number. Moreover, if $M_{1}$ and $M_{2}$ arise from Sunada's construction, then it follows from Proposition 1.4 that the numerator and denominator of this rational number are only divisible by prime divisors of $\# G$. Like in the case of torsion, it would be interesting to understand the quotient of regulators in each degree separately. In this direction we have the following result, which we now state for arbitrary $G$-relations (see Definition 2.3). Below, for a group $U$ we write $U^{\mathrm{ab}}=U /[U, U]$ where $[U, U]$ denotes the derived subgroup of $U$.

Theorem 1.7. Let $X \rightarrow Y$ be a $G$-covering of closed oriented Riemannian $d$-manifolds, and let $\sum_{j} n_{j} U_{j}, n_{j} \in \mathbb{Z}$ be a G-relation. Then:

(1) for every integer $i \geqslant 0$, the product $\prod_{j} \operatorname{Reg}_{i}\left(X / U_{j}\right)^{2 n_{j}}$ is a rational number that is a product of powers of prime divisors of $\# G$;

(2) for every $i \geq 0$, the class of $\prod_{j} \operatorname{Reg}_{i}\left(X / U_{j}\right)^{2 n_{j}}$ in $\mathbb{Q}^{\times} /\left(\mathbb{Q}^{\times}\right)^{2}$ only depends on the isomorphism class of the $G$-module $H_{i}(X, \mathbb{Q})$;

\footnotetext{
${ }^{1} \mathrm{~A}$ few months after submitting this paper, we established Conjecture 1.6 in 2 using techniques introduced in this paper, see in particular Proposition 1.8
} 
(3) if $p$ is a prime number that does not divide $\# U_{j}^{\mathrm{ab}}$ for any $j$, then the p-part of $\prod_{j} \operatorname{Reg}_{d-1}\left(X / U_{j}\right)^{2 n_{j}}=\prod_{j} \operatorname{Reg}_{1}\left(X / U_{j}\right)^{-2 n_{j}}$ only depends on the isomorphism class of the $G$-module $H_{d-1}\left(X, \mathbb{Z}_{(p)}\right)$.

The representation theoretic invariant that appears in parts (2) and (3) of the theorem was first introduced by Dokchitser-Dokchitser in the context of regulators of elliptic curves (see e.g. [13]), and is called the regulator constant of the representation with respect to the given $G$-relation.

We actually relate the quotients of regulators to regulator constants in greater generality, namely without the hypothesis on $p$ in part (3) of Theorem 1.7. But in general, there is an "error term", which measures the failure of $G$-descent for homology in the covering - see Theorem 3.11 for the precise statement, but see also Remark 3.14 .

Vignéras [37] introduced another well-known construction of isospectral manifolds, of a more arithmetic flavour. In [10, Calegari and Venkatesh study torsion homology in pairs of manifolds that are of a similar nature to the examples of Vignéras, called "Jacquet-Langlands pairs", that are not isospectral but whose spectra are closely related. They raise, in [10, Section 7.10], the question of giving algebraic interpretations to the quotients of torsion homology and regulators separately, analogously to Theorem 1.7 . It would also be interesting to investigate the same type of questions in the context of the Vignéras examples.

In Section 6, we give examples of how Theorem 1.7 allows one to deduce concrete information about the $\mathbb{Q}[G]$-module structure of $H_{1}(X, \mathbb{Q})$ from the torsion homology of quotients of $X$. It also allows one to formulate a possible representation theoretic line of attack on Conjecture 1.6, such as the following result, which will be proved as Corollary 4.4.

Proposition 1.8. Let $p$ be an odd prime, let $G=\mathrm{GL}_{2}\left(\mathbb{F}_{p}\right)$, and define the subgroups

$$
B=\left(\begin{array}{cc}
\mathbb{F}_{p}^{\times} & \mathbb{F}_{p} \\
0 & \mathbb{F}_{p}^{\times}
\end{array}\right), \quad U=\left(\begin{array}{cc}
\left(\mathbb{F}_{p}^{\times}\right)^{2} & \mathbb{F}_{p} \\
0 & \mathbb{F}_{p}^{\times}
\end{array}\right)
$$

of $G$. Suppose that there exists a $G$-covering $X \rightarrow Y$ of closed hyperbolic 3manifolds such that the difference of the Betti numbers $b_{1}(X / U)-b_{1}(X / B)$ is odd. Then Conjecture 1.6 holds for $p$.

To fully appreciate the representation theoretic nature of Proposition 1.8 , see the full statement of Corollary 4.4 .

The structure of the paper is as follows. In Section 2 we recall the formalism of Burnside groups, representation groups, and $G$-relations, and the definition of regulator constants. In Section 3 we investigate the behaviour of homological invariants of Riemannian manifolds in $G$-relations, and prove Proposition 1.4 and Theorem 1.7. In Section 4 we compute the regulator constants of the rational representations of $\mathrm{GL}_{2}\left(\mathbb{F}_{p}\right)$ for odd primes $p$, and of $(\mathbb{Z} / 8 \mathbb{Z}) \rtimes(\mathbb{Z} / 8 \mathbb{Z})^{\times}$, with respect to certain $G$-relations. These computations will allow us to deduce concrete information about the $\mathbb{Q}[G]$-module structure of the rational homology of $G$-coverings from the torsion homology of intermediate coverings. Together with Theorem 3.11, these calculations will also imply Proposition 1.8 . We prove Proposition 1.5 by a direct computer search, and Section 5 is devoted to the methods and algorithms used to that 
end. In Section 6 we have collected some interesting examples, illustrating the various phenomena that we investigate here.

All our Riemannian manifolds will be assumed to be finite-dimensional, oriented, and closed. By an automorphism of a Riemannian manifold we mean an orientation preserving diffeomorphism from the manifold to itself that is a local isometry. By a hyperbolic 3-manifold, we mean a quotient of the hyperbolic 3 -space by a discrete subgroup of orientation preserving isometries. If $p$ is a prime number, we will write $\mathbb{Z}_{(p)}$ for the localisation of $\mathbb{Z}$ at $p$, i.e. the subring $\left\{\frac{a}{b}: p \nmid b\right\}$ of $\mathbb{Q}$. For a rational number $x=p^{n} \frac{a}{b}$, with $n \in \mathbb{Z}$ and $p \nmid a b$, the $p$-adic valuation of $x$ is defined to be $\operatorname{ord}_{p}(x)=n$. When $M$ is a $\mathbb{Z}$-module, we write $M_{\text {tors }}$ for the torsion submodule of $M$ and $M^{\mathrm{fr}}=M / M_{\mathrm{tors}}$ for the torsion-free quotient of $M$.

Acknowledgements. We would like to thank Nicolas Bergeron, Nathan Dunfield, Derek Holt, Emilio Lauret, and Karen Vogtmann for helpful discussions, and the anonymous referee for suggestions that improved the exposition.

\section{2. $G$-RElations And Regulator COnstants}

We recall some standard definitions, for which we refer to [12, Ch. 11]. By "module" we will always mean a finitely generated left module.

Definition 2.1. Let $G$ be a finite group. The Burnside group of $G$ is the free abelian group on isomorphism classes of transitive $G$-sets.

The set of transitive $G$-sets is in bijection with the set of conjugacy classes of subgroups of $G$ via the map that assigns to the subgroup $U$ the set of cosets $G / U$. Using this identification, we will represent elements of the Burnside group as formal sums $\sum_{j} n_{j} U_{j}$, where $n_{j} \in \mathbb{Z}$ and $U_{j} \leq G$.

Definition 2.2. Let $R$ be a domain. The representation group of $G$ over $R$ is the free abelian group on isomorphism classes of $R$-free indecomposable $R[G]$-modules, where $R[G]$ is the group ring of $G$ over $R$.

Let $G$ be a finite group and $R$ be a domain. We write $\mathbf{1}$ for the free $R$-module of rank 1 with trivial $G$-action. We have a natural group homomorphism $\Psi_{R[G]}$ from the Burnside group of $G$ to the representation group of $G$ over $R$, which sends a $G$-set $X$ to the permutation module $R[X]$ with an $R$-basis indexed by the elements of $X$, and with the $R$-linear $G$-action given by permutations of the basis.

Definition 2.3. Let $R$ be a domain. An $R[G]$-relation is an element of the kernel of $\Psi_{R[G]}$. A $\mathbb{Q}[G]$-relation will be referred to simply as a $G$-relation.

Example 2.4. Let $p$ be a prime, and let

$$
G=\left\langle\sigma, \tau: \sigma^{p}=\tau^{2}=\mathrm{id}, \tau \sigma \tau=\sigma^{-1}\right\rangle
$$

be a dihedral group of order $2 p$. Then $\Theta=1-2 C_{2}-C_{p}+2 G$ is a $G$-relation. Concretely, this means that there is an isomorphism of linear permutation representations of $G$ over $\mathbb{Q}$,

$$
\mathbb{Q}[G / 1] \oplus \mathbb{Q}[G / G]^{\oplus 2} \cong \mathbb{Q}\left[G / C_{2}\right]^{\oplus 2} \oplus \mathbb{Q}\left[G / C_{p}\right] .
$$


Moreover, for every prime $q \neq p$, the relation $\Theta$ is in fact a $\mathbb{Z}_{(q)}[G]$-relation, but it is not a $\mathbb{Z}_{(p)}[G]$-relation - see [1, Proof of Proposition 3.9].

Example 2.5. Let $G$ be the affine linear group over $\mathbb{Z} / 8 \mathbb{Z}$, i.e. the group of linear transformations $T_{a, b}: x \mapsto a x+b$ of $\mathbb{Z} / 8 \mathbb{Z}$, where $a \in(\mathbb{Z} / 8 \mathbb{Z})^{\times}$ and $b \in \mathbb{Z} / 8 \mathbb{Z}$. Consider the subgroups $U_{1}=\left\langle T_{a, 0}: a \in(\mathbb{Z} / 8 \mathbb{Z})^{\times}\right\rangle$and $U_{2}=\left\langle T_{-1,0}, T_{3,4}\right\rangle$. The group $G$ is isomorphic to the semi-direct product $\mathbb{Z} / 8 \mathbb{Z} \rtimes(\mathbb{Z} / 8 \mathbb{Z})^{\times}$, and the subgroups $U_{1}$ and $U_{2}$ are both isomorphic to $C_{2} \times C_{2}$. Then $U_{1}-U_{2}$ is a $\mathbb{Z}_{(p)}[G]$-relation for every odd prime $p$, but is not a $\mathbb{Z}_{(2)}[G]$-relation, as can be deduced from a direct character computation and Lemma 2.7 below.

Example 2.6. Let $p$ be an odd prime, and let $G=\mathrm{GL}_{2}\left(\mathbb{F}_{p}\right)$. Consider the two subgroups

$$
U_{1}=\left(\begin{array}{cc}
\mathbb{F}_{p}^{\times} & \mathbb{F}_{p} \\
0 & \left(\mathbb{F}_{p}^{\times}\right)^{2}
\end{array}\right), \quad U_{2}=\left(\begin{array}{cc}
\left(\mathbb{F}_{p}^{\times}\right)^{2} & \mathbb{F}_{p} \\
0 & \mathbb{F}_{p}^{\times}
\end{array}\right) .
$$

Then for every prime $q, U_{1}-U_{2}$ is a $\mathbb{Z}_{(q)}[G]$-relation if and only if $q \neq p$.

Observe that $U_{1} \cap U_{2}$ contains the subgroup $N=\left\{\left(\begin{array}{cc}a & 0 \\ 0 & a\end{array}\right): a \in\left(\mathbb{F}_{p}^{\times}\right)^{2}\right\}$, which is central in $G$. Set $\bar{G}=G / N$, and $\bar{U}_{j}=U_{j} / N$ for $j=1$ and 2 . We then get the $\bar{G}$-relation $\bar{U}_{1}-\bar{U}_{2}$. Moreover, the triple $\left(\bar{G}, \bar{U}_{1}, \bar{U}_{1}\right)$ minimises the index $\left(\Gamma: S_{1}\right)$ among all triples $\left(\Gamma, S_{1}, S_{2}\right)$, where $\Gamma$ is a finite group, and $S_{1}-S_{2}$ is a $\Gamma$-relation that is not a $\mathbb{Z}_{(p)}[\Gamma]$-relation (see [34]).

Lemma 2.7. Let $G$ be a finite group, let $p$ be a prime number not dividing $\# G$, and let $\Theta$ be an element of the Burnside group of $G$. Then the following are equivalent:

(1) $\Theta$ is a $\mathbb{C}[G]$-relation;

(2) $\Theta$ is a $\mathbb{Q}[G]$-relation;

(3) $\Theta$ is a $\mathbb{Z}_{(p)}[G]$-relation;

(4) $\Theta$ is an $\mathbb{F}_{p}[G]$-relation.

Proof. The equivalence of (1) and (2) follows from the fact that if $\rho_{1}$ and $\rho_{2}$ are $\mathbb{Q}[G]$-modules, then $\operatorname{dim}_{\mathbb{Q}} \operatorname{Hom}\left(\rho_{1}, \rho_{2}\right)=\operatorname{dim}_{\mathbb{C}} \operatorname{Hom}\left(\mathbb{C} \otimes_{\mathbb{Q}} \rho_{1}, \mathbb{C} \otimes_{\mathbb{Q}} \rho_{2}\right)$ (see [31, §2e]). The remaining equivalences are proven in [3, Chapter 5].

Notation 2.8. Let $f$ be any function on the set of conjugacy classes of subgroups of a finite group $G$ with values in an abelian group $A$. Then $f$ extends to a unique group homomorphism from the Burnside group of $G$ to $A$, defined by $f\left(\sum_{j} n_{j} U_{j}\right)=\prod_{j} f\left(U_{j}\right)^{n_{j}}$, where $U_{j}$ are subgroups of $G$ and $n_{j} \in \mathbb{Z}$.

Let $S$ be a subring of $\mathbb{R}$ that is a PID. The main examples we are thinking of are $\mathbb{Z}$, the localisation $\mathbb{Z}_{(p)}$ where $p$ is a prime, and $\mathbb{Q}$. Given an $S[G]$ module $A$, let $A_{S \text {-tors }}$ denote the $S[G]$-submodule consisting of $S$-torsion

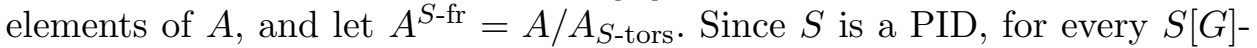
module $A$, the quotient $A^{S \text {-fr }}$ is an $S$-free $S[G]$-module. Moreover, $\mathbb{R} \otimes_{S} A$ is self-dual, i.e. it is isomorphic to the $\mathbb{R}[G]$-module $\operatorname{Hom}_{S}(A, \mathbb{R})$. Thus there exists an $\mathbb{R}$-valued $S$-bilinear $G$-invariant non-degenerate pairing on $A^{S \text {-fr }}$. From now on, we will just say pairing on $A$ when we mean an $\mathbb{R}$-valued $S$-bilinear $G$-invariant pairing on $A$ that is non-degenerate on $A^{S \text {-fr }}$. 
Definition 2.9. Let $G$ be a finite group, let $S$ a subring of $\mathbb{R}$ that is a PID, let $R$ be a subring of $\mathbb{Q}$, and let $A$ be an $S[G]$-module. Let $\langle\cdot, \cdot\rangle$ be a pairing on $A$, and let $\Theta=\sum_{j} n_{j} U_{j}$ be an $R[G]$-relation. The regulator constant of $A$ with respect to $\Theta$ is defined as

$$
\mathcal{C}_{\Theta}(A)=\prod_{j} \operatorname{det}\left(\frac{1}{\# U_{j}}\langle\cdot, \cdot\rangle \mid\left(A^{U_{j}}\right)^{S-\mathrm{fr}}\right)^{n_{j}} \in \mathbb{R}^{\times} /\left(S^{\times}\right)^{2},
$$

where the $j$-th determinant is computed with respect to any $S$-basis on $\left(A^{U_{j}}\right)^{S \text {-fr }}$. The class in $\mathbb{R}^{\times} /\left(S^{\times}\right)^{2}$ of each determinant is independent of the choice of basis.

Theorem 2.10. The value of $\mathcal{C}_{\Theta}(A)$ is independent of the pairing $\langle\cdot, \cdot\rangle$ on $A$. Proof. See [13, Theorem 2.17].

It follows from Theorem 2.10 that if $S=\mathbb{Z}$, then $\mathcal{C}_{\Theta}(A) \in \mathbb{Q}^{\times}$is welldefined. Indeed, $\left(\mathbb{Z}^{\times}\right)^{2}=\{1\}$, and the pairing can always be chosen to take values in $\mathbb{Q}$. Similarly, if $S=\mathbb{Z}_{(p)}$, then $\operatorname{ord}_{p}\left(\mathcal{C}_{\Theta}(A)\right) \in \mathbb{Z}$ is well-defined, while if $S=\mathbb{Q}$, then $\mathcal{C}_{\Theta}(A)$ defines a class in $\mathbb{Q}^{\times} /\left(\mathbb{Q}^{\times}\right)^{2}$.

Corollary 2.11. Let $A_{1}, A_{2}$ be $S[G]$-modules, and $\Theta$ a G-relation. Then $\mathcal{C}_{\Theta}\left(A_{1} \oplus A_{2}\right)=\mathcal{C}_{\Theta}\left(A_{1}\right) \mathcal{C}_{\Theta}\left(A_{2}\right)$.

Proof. We can choose the pairing on $A_{2} \oplus A_{2}$ so that the direct summands are orthogonal to each other, making all the matrices block diagonal.

Example 2.12. Let $G$ be a finite group, and let $\Theta=\sum_{j} n_{j} U_{j}$ be a $G$ relation. Then $\mathcal{C}_{\Theta}(\mathbf{1})=\prod_{j} \# U_{j}^{-n_{j}}$.

Lemma 2.13. Let $G$ be a finite group and let $\Theta=\sum_{j} n_{j} U_{j}$ be a $G$-relation. Then we have $\sum_{j} n_{j}=0$.

Proof. By definition, the virtual representation $\bigoplus_{j} \mathbb{C}\left[G / U_{j}\right]^{\oplus n_{j}}$ is zero. The result follows by taking the inner product with the trivial character of $G$.

Proposition 2.14. Let $G$ be a finite group, and let $\Theta=\sum_{j} n_{j} U_{j}$ be a $G$ relation. Let $A$ be a $\mathbb{Q}[G]$-module that has no simple summand in common with any $\mathbb{Q}\left[G / U_{j}\right]$. Then $\mathcal{C}_{\Theta}(A)=1$.

Proof. See [13, Lemma 2.26].

Let $G$ be a finite group, and $D$ a subgroup. The operation on $G$-sets of restricting the action of $G$ to $D$ extends linearly to a restriction map on the Burnside groups, and induces a restriction map on relations. With respect to the bases of the Burnside groups given by transitive $G$-sets, respectively transitive $D$-sets, the restriction map is given by Mackey's formula:

$$
\operatorname{Res}_{D}^{G} U=\sum_{x \in U \backslash G / D} D \cap x^{-1} U x .
$$

We have the following form of Frobenius reciprocity for regulator constants.

Proposition 2.16. Let $G$ be a finite group, let $D$ be a subgroup, let $A$ be an $S[D]$-module, and let $\Theta$ be a $G$-relation. Then we have

$$
\mathcal{C}_{\Theta}\left(\operatorname{Ind}_{D}^{G} A\right)=\mathcal{C}_{\operatorname{Res}_{D}^{G} \Theta}(A) .
$$

Proof. See [13, Proposition 2.45]. 


\section{TORSION HOMOLOGY AND REGULATORS IN RELATIONS}

In this section, we investigate the quotients of torsion homology and of regulators of Riemannian manifolds arising from $G$-relations. We begin by recalling some basic definitions and fixing the notation. The reader is referred to [32] for the details.

Definition 3.1. If $V, W$ are two abelian groups equipped with $\mathbb{R}$-valued bilinear forms $\langle\cdot, \cdot\rangle_{V}$, respectively $\langle\cdot, \cdot\rangle_{W}$, a similitude from $V$ to $W$ with factor ${ }^{2} \lambda \in \mathbb{R}$ is a homomorphism $f: V \rightarrow W$ such that $\left\langle f\left(v_{1}\right), f\left(v_{2}\right)\right\rangle_{W}=$ $\lambda\left\langle v_{1}, v_{2}\right\rangle_{V}$ for all $v_{1}, v_{2} \in V$. An isometry is a similitude with factor 1 .

Notation 3.2. Let $X$ be a $d$-dimensional Riemannian manifold, and let $i \geqslant 0$ be an integer.

i. Harmonic forms. The Laplace-de Rham operator acts on the differential $i$-forms on $X$, and we let $\mathcal{H}^{i}(X)$ denote the space of differential $i$-forms that lie in the kernel of that operator, the space of harmonic $i$-forms. The Riemannian metric on $X$ induces a canonical inner product on $\mathcal{H}^{i}(X)$, which induces a canonical isomorphism between $\mathcal{H}^{i}(X)$ and the $\mathbb{R}$-linear dual $\mathcal{H}^{i}(X)^{\vee}=\operatorname{Hom}\left(\mathcal{H}^{i}(X), \mathbb{R}\right)$. Explicitly, the inner product on $\mathcal{H}^{i}(X)$ is given by

$$
\left(\omega_{1}, \omega_{2}\right)_{X}^{i}=\int_{X} \omega_{1} \wedge * \omega_{2},
$$

where $\omega_{1}, \omega_{2} \in \mathcal{H}^{i}(X)$, and where $*$ denotes the Hodge star operator $*: \mathcal{H}^{i}(X) \rightarrow \mathcal{H}^{d-i}(X)$ (see [32, §1.2.3]). We equip $\mathcal{H}^{i}(X)^{\vee}$ with the inner product induced by the one on $\mathcal{H}^{i}(X)$, i.e. the unique inner product that makes the map $\omega \mapsto(\cdot, \omega)_{X}^{i}$ an isometry.

ii. Regulators. We have a homomorphism

$$
h_{X}^{i}: H_{i}(X, \mathbb{Z}) \rightarrow \mathcal{H}^{i}(X)^{\vee}
$$

given by $h_{X}^{i}(\gamma)=\left(\omega \mapsto \int_{\gamma} \omega\right)$. It follows from the Hodge Theorem ([32, Theorem 1.45]) and de Rham's Theorem that $\operatorname{ker} h_{X}^{i}=H_{i}(X, \mathbb{Z})_{\text {tors }}$ and that the image of $h_{X}^{i}$ spans $\mathcal{H}^{i}(X)^{\vee}$ over $\mathbb{R}$. The $i$-th regulator $\operatorname{Reg}_{i}(X)$ of $X$ is defined as the covolume of $h_{X}^{i}\left(H_{i}(X, \mathbb{Z})\right)$ with respect to the inner product on $\mathcal{H}^{i}(X)^{\vee}$.

Let $\langle\cdot, \cdot\rangle_{X}^{i}: H_{i}(X, \mathbb{Z}) \otimes_{\mathbb{Z}} H_{i}(X, \mathbb{Z}) \rightarrow \mathbb{R}$ denote the pullback via $h_{X}^{i}$ of the pairing on $\mathcal{H}^{i}(X)^{\vee}$, i.e. the unique pairing on $H_{i}(X, \mathbb{Z})$ that makes $h_{X}^{i}$ an isometry.

iii. Poincaré duality. By Poincaré duality, the map

$$
D_{X}: H^{d-i}(X, \mathbb{Z}) \rightarrow H_{i}(X, \mathbb{Z}),
$$

given by cap product with the fundamental class of $X$ is an isomorphism. It follows that $\operatorname{Reg}_{i}(X)=\operatorname{Reg}_{d-i}(X)^{-1}$.

iv. Actions of automorphism groups. Let $G$ be a group of automorphisms of $X$. Then $G$ acts on the left by linear transformations on $H_{i}(X, \mathbb{Z})$ via pushforward, $\gamma \mapsto \sigma_{*} \gamma$ for $\sigma \in G$ and $\gamma \in H_{i}(X, \mathbb{Z})$. Also, $G$ acts linearly on the right on $\mathcal{H}^{i}(X)$ via pullback, $\omega \mapsto \sigma^{*} \omega$ for $\sigma \in G$ and $\omega \in \mathcal{H}^{i}(X)$, which induces the dual action on the left on $\mathcal{H}^{i}(X)^{\vee}$. It

\footnotetext{
${ }^{2}$ The similitude factor is sometimes defined to be the square root of our convention.
} 
follows from the adjointness of pullbacks and pushforwards that the map $h_{X}^{i}$ is a homomorphism of left $G$-modules. Moreover, recall that our automorphisms are assumed to be orientation preserving, so the Poincaré duality map $D_{X}$ is an isomorphism of $G$-modules.

Lemma 3.3. Let $X$ be a Riemannian manifold. Then we have $\operatorname{det}\langle\cdot, \cdot\rangle_{X}^{i}=$ $\operatorname{Reg}_{i}(X)^{2}$, where the left hand side is computed with respect to any $\mathbb{Z}$-basis of $H_{i}(X, \mathbb{Z})^{\mathrm{fr}}$. In particular, the pairing $\langle\cdot, \cdot\rangle_{X}^{i}$ is non-degenerate on $H_{i}(X, \mathbb{Z})^{\mathrm{fr}}$.

Proof. The claimed equality is the expression of the covolume of a lattice in terms of the determinant of its Gram matrix.

Remark 3.4. Explicitly, let $\omega_{1}, \ldots, \omega_{r}$ be an orthonormal basis of $\mathcal{H}^{i}(X)$, and let $\left(\gamma_{j}\right)$ be a $\mathbb{Z}$-basis of $H_{i}(X, \mathbb{Z})^{\mathrm{fr}}$. Then we have

$$
\operatorname{Reg}_{i}(X)=\left|\operatorname{det} \int_{\gamma_{j}} \omega_{k}\right|,
$$

and for all $\gamma, \gamma^{\prime} \in H_{i}(X, \mathbb{Z})$, we have $\left\langle\gamma, \gamma^{\prime}\right\rangle_{X}^{i}=\sum_{k=1}^{r}\left(\int_{\gamma} \omega_{k} \cdot \int_{\gamma^{\prime}} \omega_{k}\right)$.

We now prove Proposition 1.4 as a consequence of the following result.

Theorem 3.5. Let $X \rightarrow Y$ be a finite $G$-covering of Riemannian manifolds, and let $\Theta=\sum_{j} n_{j} U_{j}-\sum_{k} n_{k}^{\prime} U_{k}^{\prime}$ be a $\mathbb{Z}_{(p)}[G]$-relation, where $n_{j}$ and $n_{k}^{\prime}$ are positive integers. Then for every integer $i \geqslant 0$, we have

$$
\bigoplus_{j} H_{i}\left(X / U_{j}, \mathbb{Z}\right)\left[p^{\infty}\right]^{n_{j}} \cong \bigoplus_{k} H_{i}\left(X / U_{k}^{\prime}, \mathbb{Z}\right)\left[p^{\infty}\right]^{n_{k}^{\prime}} .
$$

In particular we have $\operatorname{ord}_{p}\left(\# H_{i}(X / \Theta, \mathbb{Z})_{\text {tors }}\right)=0$ (see Notation 2.8).

Proof. Let $U$ be any subgroup of $G$. Then the $p^{\infty}$-torsion of $H_{i}(X / U, \mathbb{Z})$ is isomorphic to the torsion subgroup of $H_{i}(X / U, \mathbb{Z}) \otimes \mathbb{Z}_{(p)} \cong H_{i}\left(X / U, \mathbb{Z}_{(p)}\right)$. By definition of homology with local coefficients (see [16, $\S 3 . \mathrm{H}]$ ), we have $H_{i}\left(X / U, \mathbb{Z}_{(p)}\right) \cong H_{i}\left(X / G, \mathbb{Z}_{(p)}[G / U]\right)$. Since homology with local coefficients is additive in direct sums of modules, the result follows.

Corollary 3.6. Let $X \rightarrow Y$ and $G$ be as in Theorem 3.5, let $p$ be a prime number that does not divide $\# G$, and let $\Theta$ be a G-relation. Then

$$
\bigoplus_{j} H_{i}\left(X / U_{j}, \mathbb{Z}\right)\left[p^{\infty}\right]^{n_{j}} \cong \bigoplus_{k} H_{i}\left(X / U_{k}^{\prime}, \mathbb{Z}\right)\left[p^{\infty}\right]^{n_{k}^{\prime}} .
$$

Proof. This is a direct consequence of Theorem 3.5 and Lemma 2.7.

The rest of the section is devoted to the behaviour of regulators in $G$ relations. In particular, we will prove Theorem 1.7 .

Lemma 3.7. Let $X \rightarrow Y$ be a finite $G$-covering of Riemannian manifolds of dimension $d$, and let $\Theta=\sum_{j} n_{j} U_{j}$ be a G-relation. Then

$$
\operatorname{Reg}_{d}(X / \Theta)^{2}=\operatorname{Reg}_{0}(X / \Theta)^{-2}=\mathcal{C}_{\Theta}(\mathbf{1}) .
$$

Proof. For all $U \leq G$, we have $\operatorname{Reg}_{d}(X / U)^{2}=\operatorname{Reg}_{0}(X / U)^{-2}=\frac{1}{\# U} \operatorname{vol}(X)$. It follows that

$$
\operatorname{Reg}_{d}(X / \Theta)^{2}=\operatorname{Reg}_{0}(X / \Theta)^{-2}=\left(\prod_{j} \frac{1}{\# U_{j}^{n_{j}}}\right) \cdot \operatorname{vol}(X)^{\left(\sum_{j} n_{j}\right)}=\mathcal{C}_{\Theta}(\mathbf{1}),
$$


where the last equality follows from Example 2.12 and Lemma 2.13

Lemma 3.8. Let $f: X \rightarrow Y$ be a finite $G$-covering of Riemannian manifolds, and let $i \geqslant 0$ be an integer. Then the following maps are similitudes with factor \#G:

(1) the pullback map $f^{*}: \mathcal{H}^{i}(Y) \rightarrow \mathcal{H}^{i}(X)^{G}$;

(2) its dual $\left(f^{*}\right)^{\vee}:\left(\mathcal{H}^{i}(X)^{G}\right)^{\vee} \rightarrow \mathcal{H}^{i}(Y)^{\vee}$;

(3) the pushforward map $f_{*}: H_{i}(X, \mathbb{Z})^{G} \rightarrow H_{i}(Y, \mathbb{Z})$;

(4) any map $g: H_{i}(Y, \mathbb{Z}) \rightarrow H_{i}(X, \mathbb{Z})^{G}$ satisfying $f_{*} g=\# G \cdot \operatorname{id}_{H_{i}(Y, \mathbb{Z})}$.

Proof. (1) Let $[X]$ and $[Y]$ denote the fundamental class on $X$, respectively on $Y$. Then $f_{*}[X]=\# G \cdot[Y]$, so for all $\omega_{1}, \omega_{2} \in \mathcal{H}^{i}(Y)$, we have

$$
\begin{aligned}
\left(f^{*} \omega_{1}, f^{*} \omega_{2}\right)_{X}^{i} & =\int_{X} f^{*} \omega_{1} \wedge f^{*} * \omega_{2}=\int_{X} f^{*}\left(\omega_{1} \wedge * \omega_{2}\right) \\
& =\# G \cdot \int_{Y} \omega_{1} \wedge * \omega_{2}=\# G \cdot\left(\omega_{1}, \omega_{2}\right)_{Y}^{i} .
\end{aligned}
$$

(2) The assertion immediately follows from part (1), from the fact that $f^{*}$ is an isomorphism, and from the definition of the inner product on the dual space, see Notation 3.2 .

(3) By Notation 3.2 iv. the map $h_{X}^{i}$ sends $H_{i}(X, \mathbb{Z})^{G}$ to $\left(\mathcal{H}^{i}(X)^{G}\right)^{\vee}$, and it follows from the adjointness of pullbacks and pushforwards that the diagram

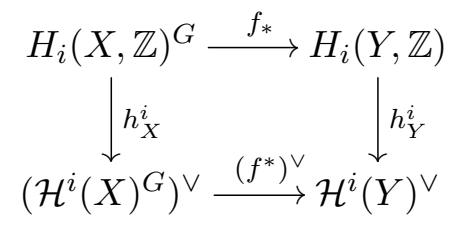

is commutative. Note that $\left(\mathcal{H}^{i}(X)^{G}\right)^{\vee}$ is canonically isomorphic, as an inner product space, to the $G$-coinvariants $\left(\mathcal{H}^{i}(X)^{\vee}\right)_{G}$, which in turn is canonically isomorphic to $\left(\mathcal{H}^{i}(X)^{\vee}\right)^{G}$. This shows that $h_{X}^{i}: H_{i}(X, \mathbb{Z})^{G} \rightarrow$ $\left(\mathcal{H}^{i}(X)^{G}\right)^{\vee}$ is an isometry. By definition, $h_{Y}^{i}$ is also an isometry, and by part (2), $\left(f^{*}\right)^{\vee}$ is a similitude with factor $\# G$. It follows that $f_{*}$ is also a similitude with factor $\# G$.

(4) By part (3), the map $f_{*}$ is a similitude with factor $\# G$, and the claim immediately follows.

Lemma 3.9. Let $X \rightarrow Y$ be a finite $G$-covering of Riemannian manifolds, and let $i \geqslant 0$ be an integer. Then the composition

$$
g_{i}: H_{i}(Y, \mathbb{Z}) \stackrel{D_{Y}^{-1}}{\longrightarrow} H^{d-i}(Y, \mathbb{Z}) \stackrel{f^{*}}{\longrightarrow} H^{d-i}(X, \mathbb{Z})^{G} \stackrel{D_{X}}{\longrightarrow} H_{i}(X, \mathbb{Z})^{G}
$$

satisfies $f_{*} g_{i}=\# G \cdot \operatorname{id}_{H_{i}(Y, \mathbb{Z})}$.

Proof. The pushforward of the fundamental class of $X$ is equal to \#G times the fundamental class of $Y$. It therefore follows from the naturality of the cap product that $\# G \cdot D_{Y}=f_{*} D_{X} f^{*}$, which proves the result.

Lemma 3.10. Let $X \rightarrow Y$ be a finite $G$-covering of Riemannian manifolds, and let $i \geqslant 0$ be an integer. Let $g: H_{i}(Y, \mathbb{Z}) \rightarrow H_{i}(X, \mathbb{Z})^{G}$ be any map 
satisfying $f_{*} g=\# G \cdot \operatorname{id}_{H_{i}(Y, \mathbb{Z})}$, and let

$$
\lambda_{i}(X, G, g)=\left(H_{i}(X, \mathbb{Z})^{G}: g\left(H_{i}(Y, \mathbb{Z})\right)+H_{i}(X, \mathbb{Z})_{\text {tors }}^{G}\right) .
$$

Then $\lambda_{i}(X, G, g)$ is finite and is independent of $g$.

Proof. The quantity $\lambda_{i}(X, G, g)$ is finite since $g \otimes \mathbb{Q}: H_{i}(Y, \mathbb{Q}) \rightarrow H_{i}(X, \mathbb{Q})^{G}$ is an isomorphism. If $g^{\prime}$ is another map satisfying the conditions of the lemma, then $f_{*}\left(g-g^{\prime}\right)=0$. It follows from this, and from the fact that $f_{*} \otimes \mathbb{Q}$ is an isomorphism, that $\operatorname{Im}\left(g-g^{\prime}\right) \subset \operatorname{ker} f_{*} \subset H_{i}(X, \mathbb{Z})_{\text {tors }}^{G}$. Therefore

$$
g\left(H_{i}(Y, \mathbb{Z})\right)+H_{i}(X, \mathbb{Z})_{\text {tors }}^{G}=g^{\prime}\left(H_{i}(Y, \mathbb{Z})\right)+H_{i}(X, \mathbb{Z})_{\text {tors }}^{G},
$$

which proves the lemma.

Given a finite $G$-covering $X \rightarrow Y$ and $i$ as in Lemma 3.10, we define $\lambda_{i}(X, G)=\lambda_{i}(X, G, g)$ for any $g$ satisfying the hypotheses of the lemma.

Theorem 3.11. Let $X \rightarrow Y$ be a G-covering of Riemannian manifolds, let $\Theta=\sum_{j} n_{j} U_{j}$ be a G-relation (see Definition 2.3), and let $i \geqslant 0$ be an integer. Then we have

$$
\operatorname{Reg}_{i}(X / \Theta)^{2}=\mathcal{C}_{\Theta}\left(H_{i}(X, \mathbb{Z})\right) \cdot \lambda_{i}(X, \Theta)^{2} .
$$

Proof. By Lemma 3.3 , the pairing $\langle\cdot, \cdot\rangle_{X}^{i}$ on $H_{i}(X, \mathbb{Z})$ is non-degenerate on the quotient modulo torsion. Moreover, by Lemma 3.8 (3) applied to $f=\sigma$ for each $\sigma \in G$, this pairing is $G$-invariant. For any $U_{j} \leq G$, let $g$ be any map satisfying the conditions of Lemma 3.10 for the covering $X \rightarrow X / U_{j}$. Then we have

$$
\begin{aligned}
& \operatorname{det}(\left.\frac{1}{\# U_{j}}\langle\cdot, \cdot\rangle_{X}^{i} \mid H_{i}(X, \mathbb{Z})^{U_{j}^{\mathrm{fr}}}\right) \\
& \quad=\operatorname{det}\left(\frac{1}{\# U_{j}}\langle\cdot, \cdot\rangle_{X}^{i} \mid g\left(H_{i}\left(X / U_{j}, \mathbb{Z}\right)\right)^{\mathrm{fr}}\right) / \lambda_{i}\left(X, U_{j}\right)^{2} \\
& \quad=\operatorname{det}\left(\langle\cdot, \cdot\rangle_{X / U_{j}}^{i} \mid H_{i}\left(X / U_{j}, \mathbb{Z}\right)^{\mathrm{fr}}\right) / \lambda_{i}\left(X, U_{j}\right)^{2} \\
& \quad=\operatorname{Reg}_{i}\left(X / U_{j}\right)^{2} / \lambda_{i}\left(X, U_{j}\right)^{2},
\end{aligned}
$$

where the second equality follows from Lemma 3.8(4), and the last one from Lemma 3.3. This proves the theorem, by taking the product over $j$ corresponding to $\Theta$.

Corollary 3.12. Let $X \rightarrow Y$ be a G-covering of Riemannian manifolds, let $\Theta=\sum_{j} n_{j} U_{j}$ be a $G$-relation, and let $i \geqslant 0$ be an integer. Then we have $\operatorname{Reg}_{i}(X / \Theta)^{2} \equiv \mathcal{C}_{\Theta}\left(H_{i}(X, \mathbb{Q})\right)\left(\bmod \left(\mathbb{Q}^{\times}\right)^{2}\right)$.

Let $U$ be a group. The abelianisation of $U$ is the quotient $U^{\text {ab }}=U /[U, U]$, where $[U, U]=\left\langle u v u^{-1} v^{-1}: u, v \in U\right\rangle$, i.e. the maximal abelian quotient of $U$.

Corollary 3.13. Let $f: X \rightarrow Y$ be a finite $G$-covering of Riemannian dmanifolds, let $\Theta=\sum_{j} n_{j} U_{j}$ be a $G$-relation, let $p$ be a prime, and let $i \geqslant 0$ be an integer. Assume that one of the following holds:

(1) $i=d-1$ and for each subgroup $U_{j} \leq G$, the order of $U_{j}^{\mathrm{ab}}$ is not divisible by $p$

(2) for each subgroup $U_{j} \leq G$, the order of $U_{j}$ is not divisible by $p$. 
Then $\operatorname{ord}_{p}\left(\operatorname{Reg}_{i}(X / \Theta)^{2}\right)=\operatorname{ord}_{p}\left(\mathcal{C}_{\Theta}\left(H_{i}(X, \mathbb{Z})\right)\right)=\operatorname{ord}_{p}\left(\mathcal{C}_{\Theta}\left(H_{i}\left(X, \mathbb{Z}_{(p)}\right)\right)\right)$.

In particular, for all $i \geqslant 0$ the rational number $\operatorname{Reg}_{i}(X / \Theta)^{2}$ is a product of the primes dividing the order of $G$.

Proof. Let $U$ be any subgroup of $G$. Let $g_{i}=D_{X} f^{*} D_{X / U}^{-1}$ be the map of Lemma 3.9 . In case (1), the 5-term exact sequence coming from the CartanLeray spectral sequence in cohomology (see [9, Ch. VII, §7]) is

$$
0 \rightarrow H^{1}(U, \mathbb{Z}) \rightarrow H^{1}(X / U, \mathbb{Z}) \stackrel{f^{*}}{\rightarrow} H^{1}(X, \mathbb{Z})^{U} \rightarrow H^{2}(U, \mathbb{Z}) .
$$

By the universal coefficient theorem, we have $H^{2}(U, \mathbb{Z}) \cong H_{1}(U, \mathbb{Z})=U^{\mathrm{ab}}$, which has order coprime to $p$ by assumption. In case (2), the group $U$ has cohomological dimension 0 over $\mathbb{Z}_{(p)}$, since $p$ does not divide its order, so by the Cartan-Leray spectral sequence we have an isomorphism

$$
H^{d-i}\left(X / U, \mathbb{Z}_{(p)}\right) \stackrel{f^{*}}{\rightarrow} H^{d-i}\left(X, \mathbb{Z}_{(p)}\right)^{U} .
$$

In both cases, it follows that $f^{*}: H^{d-i}(X / U, \mathbb{Z}) \rightarrow H^{d-i}(X, \mathbb{Z})^{U}$ has cokernel of order coprime to $p$, and since the Poincaré duality maps $D_{X}$ and $D_{X / U}$ are isomorphisms, the cokernel of $g_{i}: H_{i}(X / U, \mathbb{Z}) \rightarrow H_{i}(X, \mathbb{Z})^{U}$ is also of order coprime to $p$. The first equality therefore follows from Theorem 3.11. For the second equality, note that firstly, $H_{i}\left(X, \mathbb{Z}_{(p)}\right) \cong H_{i}(X, \mathbb{Z}) \otimes_{\mathbb{Z}} \mathbb{Z}_{(p)}$, and secondly that for an arbitrary $\mathbb{Z}[G]$-module $A$, we have $\operatorname{ord}_{p}\left(\mathcal{C}_{\Theta}(A)\right)=$ $\operatorname{ord}_{p}\left(\mathcal{C}_{\Theta}\left(A \otimes_{\mathbb{Z}} \mathbb{Z}_{(p)}\right)\right)$.

Remark 3.14. We do not know whether the regulator quotient is a purely representation theoretic invariant in full generality without the restrictive assumptions of Corollary 3.13 , that is whether it only depends on the $\mathbb{Z}[G]$ module structure of $H_{i}(X, \mathbb{Z})$. This seems to us to be an interesting question.

\section{Some Regulator constant Calculations}

In this section, we compute the regulator constants of rational representations with respect to the relations of Examples 2.5 and 2.6.

First, let $G=\mathrm{GL}_{2}\left(\mathbb{F}_{p}\right)$. Denote by $B$ the subgroup of upper-triangular matrices in $G$, let $w=\left(\begin{array}{ll}0 & 1 \\ 1 & 0\end{array}\right)$, and let $U_{1}$, respectively $U_{2}$ be the subgroup of matrices in $B$ with square lower right, respectively upper left entry. Then $\Theta=U_{1}-U_{2}$ is the $G$-relation of Example 2.6. In this section, we compute the regulator constants of the rational irreducible representations of $G$ with respect to $\Theta$.

We begin by recalling the classification of complex representations of $G$. For any 1-dimensional representations $\mu_{1}, \mu_{2}$ of $\mathbb{F}_{p}^{\times}$, let $r_{\mu_{1}, \mu_{2}}$ be the 1dimensional representation of $B$ defined by $r_{\mu_{1}, \mu_{2}}:\left(\begin{array}{ll}a & b \\ 0 & d\end{array}\right) \mapsto \mu_{1}(a) \mu_{2}(d)$.

Proposition 4.1. The following is a classification of the complex irreducible representations of $G$ :

- 1-dimensional representations: they are all of the form $\mu$ odet, where $\mu$ is an irreducible character of $\mathbb{F}_{p}^{\times}$.

- Irreducible principal series, of dimension $p+1$ : they are the inductions $\rho\left(\mu_{1}, \mu_{2}\right)=\operatorname{Ind}_{B}^{G} r_{\mu_{1}, \mu_{2}}$, where $\mu_{1} \neq \mu_{2}$; we have $\rho\left(\mu_{1}, \mu_{2}\right) \cong$ $\rho\left(\mu_{1}^{\prime}, \mu_{2}^{\prime}\right)$ if and only if $\left\{\mu_{1}, \mu_{2}\right\}=\left\{\mu_{1}^{\prime}, \mu_{2}^{\prime}\right\}$. 
- Special representations, of dimension $p$ : they are the p-dimensional summands $\sigma(\mu)$ of $\operatorname{Ind}_{B}^{G} r_{\mu, \mu} \cong \sigma(\mu) \oplus(\mu \circ$ det $)$.

- Cuspidal representations, of dimension $p-1$ : they are the irreducible representations of $G$ that are not summands of any $\operatorname{Ind}_{B}^{G} r_{\mu_{1}, \mu_{2}}$.

Proof. See [29].

Let St denote the complex irreducible representation $\sigma(\mathbf{1})$, the Steinberg representation of $G$, and let $I$ denote the complex irreducible representation $\rho(\chi, \mathbf{1})$, where $\chi$ is the unique character of $\mathbb{F}_{p}^{\times}$of order 2 . Note that both St and $I$ are realisable over $\mathbb{Q}$, so in fact give rise to irreducible $\mathbb{Q}[G]$ representations.

Proposition 4.2. Let $\rho$ be an irreducible $\mathbb{Q}[G]$-representation. Then $\mathcal{C}_{\Theta}(\rho) \equiv p \bmod \left(\mathbb{Q}^{\times}\right)^{2}$ if $\rho \cong I$, and $\mathcal{C}_{\Theta}(\rho) \equiv 1 \bmod \left(\mathbb{Q}^{\times}\right)^{2}$ otherwise.

Proof. We have $\mathbb{C}\left[G / U_{1}\right] \cong \mathbb{C}\left[G / U_{2}\right] \cong \mathbf{1} \oplus$ St $\oplus I$. So if $\rho$ is an irreducible $\mathbb{Q}[G]$-representation that is not isomorphic to any of these three direct summands, then $\mathcal{C}_{\Theta}(\rho)=1$ by Proposition 2.14. Moreover, we have $\mathcal{C}_{\Theta}(\mathbf{1})=$ $\# U_{2} / \# U_{1}=1$ by Example 2.12

To compute $\mathcal{C}_{\Theta}(\mathrm{St})$, observe that by Corollary 2.11 and by Proposition 2.16, we have

$$
\mathcal{C}_{\Theta}(\mathrm{St})=\mathcal{C}_{\Theta}(\mathrm{St}) \mathcal{C}_{\Theta}(\mathbf{1})=\mathcal{C}_{\Theta}\left(\operatorname{Ind}_{B}^{G} \mathbf{1}\right)=\mathcal{C}_{\operatorname{Res}_{B}^{G} \Theta}(\mathbf{1}) .
$$

Let $T_{j}=U_{j} \cap T$ for $j=1,2$. An elementary calculation (or the Bruhat decomposition) shows that for $j=1$ and $2, U_{j} \backslash G / B$ has cardinality 2, with double coset representatives 1 and $w$. Moreover, we have $w^{-1} U_{1} w \cap B=T_{2}$, and $w^{-1} U_{2} w \cap B=T_{1}$. It follows from equation 2.15 that $\operatorname{Res}_{B}^{G} \Theta=$ $U_{1}+T_{2}-U_{2}-T_{1}$. We conclude from equation (4.3) and Example 2.12 that

$$
\mathcal{C}_{\Theta}(\mathrm{St})=\frac{\# U_{2} \cdot \# T_{1}}{\# U_{1} \cdot \# T_{2}}=1 \bmod \left(\mathbb{Q}^{\times}\right)^{2} .
$$

Similarly, we have

$$
\mathcal{C}_{\Theta}(I)=\mathcal{C}_{\Theta}\left(\operatorname{Ind}_{B}^{G} r_{\chi, \mathbf{1}}\right)=\mathcal{C}_{\operatorname{Res}_{B}^{G} \Theta}\left(r_{\chi, \mathbf{1}}\right) .
$$

Since $r_{\chi, \mathbf{1}}$ is 1-dimensional, the contribution from each subgroup $U$ in $\operatorname{Res}_{B}^{G} \Theta$ is 1 if $\left(r_{\chi, \mathbf{1}}\right)^{U}$ is zero, and is $(\# U)^{-1}$ otherwise. It follows that $\mathcal{C}_{\Theta}(I) \equiv$ $\# U_{2} / \# T_{2} \equiv p \bmod \left(\mathbb{Q}^{\times}\right)^{2}$.

Corollary 4.4. Let $p$ be an odd prime, let $G, B, U_{1}$, and $U_{2}$ be as above, and let $X \rightarrow Y$ be a $G$-covering of closed hyperbolic 3-manifolds. Then the following are congruent modulo 2:

(1) the $p$-adic valuation $\operatorname{ord}_{p}\left(\frac{\# H_{1}\left(X / U_{1}, \mathbb{Z}\right)_{\text {tors }}}{\# H_{1}\left(X / U_{2}, \mathbb{Z}\right)_{\text {tors }}}\right)$;

(2) the multiplicity in $H_{1}(X, \mathbb{C})$ of the irreducible $\mathbb{C}[G]$-representation $I$, as defined after Proposition 4.1:

(3) the difference of the Betti numbers $b_{1}\left(X / U_{1}\right)-b_{1}(X / B)$.

Proof. First, we show the equality between (1) and (2). For closed hyperbolic 3 -manifolds, all homology groups with integral coefficients except possibly 
the first are torsion-free. It therefore follows from equation 1.2 , Remark 3.4 . Lemma 3.7, and Example 2.12, that

$$
\frac{\# H_{1}\left(X / U_{1}, \mathbb{Z}\right)_{\text {tors }}}{\# H_{1}\left(X / U_{2}, \mathbb{Z}\right)_{\text {tors }}}=\frac{\operatorname{Reg}_{0}\left(X / U_{2}\right)^{2} \operatorname{Reg}_{1}\left(X / U_{1}\right)^{2}}{\operatorname{Reg}_{0}\left(X / U_{1}\right)^{2} \operatorname{Reg}_{1}\left(X / U_{2}\right)^{2}}=\frac{\operatorname{Reg}_{1}\left(X / U_{1}\right)^{2}}{\operatorname{Reg}_{1}\left(X / U_{2}\right)^{2}} .
$$

By Corollary 3.12, the $p$-adic valuation of the right hand side is congruent to $\operatorname{ord}_{p} \mathcal{C}_{\Theta}\left(H_{1}(X, \mathbb{Z})\right) \equiv \operatorname{ord}_{p} \mathcal{C}_{\Theta}\left(H_{1}(X, \mathbb{Q})\right)(\bmod 2)$, where $\Theta=U_{1}-U_{2}$. Recall that the right hand side of that last congruence is only well-defined modulo 2. The equality of (1) and (2) therefore follows from Corollary 2.11 and Proposition 4.2 .

We now prove the equality between (2) and (3). Below, we will identify representations of $G$ with their characters. Let $\chi$ be an arbitrary rational representation of $G$. We have $I=\operatorname{Ind}_{U_{1}}^{G} \mathbf{1}-\operatorname{Ind}_{B}^{G} \mathbf{1}$, so the multiplicity of $I$ in $\chi$ is equal to

$$
\begin{aligned}
\langle I, \chi\rangle_{G} & =\left\langle\operatorname{Ind}_{U_{1}}^{G} \mathbf{1}-\operatorname{Ind}_{B}^{G} \mathbf{1}, \chi\right\rangle_{G}=\left\langle\mathbf{1}, \operatorname{Res}_{U_{1}} \chi\right\rangle_{U_{1}}-\left\langle\mathbf{1}, \operatorname{Res}_{B} \chi\right\rangle_{B} \\
& =\operatorname{dim}_{\mathbb{Q}} \chi^{U_{1}}-\operatorname{dim}_{\mathbb{Q}} \chi^{B},
\end{aligned}
$$

where $\langle\cdot, \cdot\rangle_{U}$ denotes inner products of characters of $U$. The equality between $(2)$ and $(3)$ follows from this calculation, with $\chi=H_{1}(X, \mathbb{Q})$.

As in Example 2.6, let $N=\left\{\left(\begin{array}{cc}a & 0 \\ 0 & a\end{array}\right): a \in\left(\mathbb{F}_{p}^{\times}\right)^{2}\right\}$, let $\bar{G}=G / N, \bar{U}_{j}=U_{j} / N$ for $j=1,2$, and let $\Theta=\bar{U}_{1}-\bar{U}_{2}$, which is a $\bar{G}$-relation. There is a canonical bijection between irreducible $\mathbb{Q}[G]$-representations with kernel containing $N$ and irreducible $\mathbb{Q}[\bar{G}]$-representations, given by projecting to the quotient. For any irreducible $\mathbb{Q}[G]$-representation $\rho$ with kernel containing $N$, let $\bar{\rho}$ denote its image under this bijection.

Corollary 4.5. Let $\bar{\rho}$ be an irreducible $\mathbb{Q}[\bar{G}]$-representation. Then $\mathcal{C}_{\bar{\Theta}}(\bar{\rho}) \equiv p \bmod \left(\mathbb{Q}^{\times}\right)^{2}$ if $\bar{\rho} \cong \bar{I}$, and $\mathcal{C}_{\bar{\Theta}}(\bar{\rho}) \equiv 1 \bmod \left(\mathbb{Q}^{\times}\right)^{2}$ otherwise.

Proof. By [13, Proposition 2.45], we have $\mathcal{C}_{\bar{\Theta}}(\bar{\rho})=\mathcal{C}_{\Theta}(\rho)$. The result therefore immediately follows from Proposition 4.2 .

Now let $G$ be the group of affine linear transformations $T_{a, b}: x \mapsto a x+b$ of $\mathbb{Z} / 8 \mathbb{Z}$, where $a \in(\mathbb{Z} / 8 \mathbb{Z})^{\times}$and $b \in \mathbb{Z} / 8 \mathbb{Z}$, let $U_{1}=\left\langle T_{a, 0}: a \in(\mathbb{Z} / 8 \mathbb{Z})^{\times}\right\rangle$ and $U_{2}=\left\langle T_{-1,0}, T_{3,4}\right\rangle$, as in Example 2.5. Let $\chi$ be an irreducible faithful representation of $N=\left\langle T_{1, b}: b \in \mathbb{Z} / 8 \mathbb{Z}\right\rangle$. Then $I=\operatorname{Ind}_{N}^{G} \chi$ is a 4-dimensional irreducible $\mathbb{C}[G]$-representation, and is realisable over $\mathbb{Q}$, so it gives rise to an irreducible $\mathbb{Q}[G]$-representation. Its isomorphism class does not depend on the choice of the representation $\chi$. All the other complex irreducible representations of $G$ are also realisable over $\mathbb{Q}$, and are 1- and 2-dimensional. An explicit calculation, which we omit, gives the regulator constants of all the irreducible $\mathbb{Q}[G]$-representations with respect to $\Theta=U_{1}-U_{2}$ as follows.

Proposition 4.6. Let $\rho$ be an irreducible $\mathbb{Q}[G]$-representation. Then $\mathcal{C}_{\Theta}(\rho) \equiv 2 \bmod \left(\mathbb{Q}^{\times}\right)^{2}$ if $\rho \cong I$, and $\mathcal{C}_{\Theta}(\rho) \equiv 1 \bmod \left(\mathbb{Q}^{\times}\right)^{2}$ otherwise.

\section{Computations}

In this section, we present the computational methods used to prove Proposition 1.5. The computations were run on the Warwick number theory 
cluster. We have made the resulting data available at https://arxiv.org/ abs/1601.06821.

For each prime $p$, we look for explicit pairs of manifolds $M_{1}$ and $M_{2}$ as in Proposition 1.5. The pairs we construct all arise as intermediate coverings of $G$-coverings $X \rightarrow Y$ of closed hyperbolic 3-manifolds, for suitable finite groups $G$. We compute many such coverings, and explicitly compute the homology groups of suitable intermediate covers. There are several choices involved, which we now explain in detail.

5.1. The choice of $Y$. We only compute with arithmetic hyperbolic 3manifolds. We will recall the definitions, assuming basic facts and terminology on quaternion algebras; a standard reference is [21].

Let $\mathfrak{H}^{3}$ denote the hyperbolic 3 -space. We identify the group of orientation preserving isometries of $\mathfrak{H}^{3}$ with $\mathrm{PSL}_{2}(\mathbb{C})$ via the Poincaré extension ([21, p. 48]). For any order $\mathcal{O}$ in a quaternion algebra, let $\mathcal{O}^{1}$ denote the group of elements in $\mathcal{O}$ of reduced norm 1. A number field is called almost totally real (ATR) if it has exactly one complex place. A quaternion algebra over an ATR number field $F$ is called Kleinian if it ramifies at every real place of $F$. For any ring $R$, let $\mathcal{M}_{2}(R)$ denote the algebra of $2 \times 2$ matrices over $R$.

Definition 5.1. An arithmetic hyperbolic 3-manifold is a manifold commensurable with a quotient $\mathfrak{H}^{3} / \Gamma(\mathcal{O})$, where $\Gamma(\mathcal{O})$ arises as follows. Let $B / F$ be a Kleinian quaternion algebra over an ATR number field, and let $\iota: B \otimes_{F} \mathbb{C} \cong$ $\mathcal{M}_{2}(\mathbb{C})$ be an isomorphism induced by a complex embedding of $F$. Then define $\Gamma(\mathcal{O})=\iota\left(\mathcal{O}^{1}\right) /\{ \pm 1\} \subset \mathrm{PSL}_{2}(\mathbb{C})$.

An arithmetic hyperbolic 3-manifold has finite volume and is compact if and only if $B$ is a division algebra - see [21, Theorem 8.2.2].

Let $B / F$ be a quaternion algebra over a number field, let $\mathbb{Z}_{F}$ denote the ring of integers in $F$, and let $\mathcal{O}_{\max }$ be a maximal order in $B$. Let $\mathfrak{N}$ be an ideal of $\mathbb{Z}_{F}$ coprime to the discriminant of $B$, so that $\mathcal{O}_{\max } \otimes_{\mathbb{Z}_{F}} \mathbb{Z}_{F} / \mathfrak{N} \cong$ $\mathcal{M}_{2}\left(\mathbb{Z}_{F} / \mathfrak{N}\right)$, and let $\mathcal{O}_{0}(\mathfrak{N}) \subset \mathcal{O}_{\text {max }}$ be the preimage of the subring of uppertriangular matrices under such an isomorphism. Then $\mathcal{O}_{0}(\mathfrak{N})$ is an order in $B$.

Lemma 5.2. Let $F$ be a number field, and let $B$ be a quaternion algebra over $F$ that is a division algebra. Let $\mathcal{S}$ be the set of primes $q$ such that $F\left(\zeta_{2 q}\right)$ is isomorphic to a quadratic extension of $F$ contained in $B$, where $\zeta_{2 q}$ denotes a primitive $(2 q)$-th root of unity in an algebraic closure of $F$. For each $q \in \mathcal{S}$, and for each primitive $(2 q)$-th root of unity $\zeta$, choose a prime ideal $\mathfrak{p}_{\zeta}$ of $\mathbb{Z}_{F}$ such that the minimal polynomial of $\zeta$ is irreducible modulo $\mathfrak{p}_{\zeta}$. Let $\mathcal{P}$ be the set of such $\mathfrak{p}_{\zeta}$. Then:

(1) the set $\mathcal{S}$ is finite;

(2) for every ideal $\mathfrak{N}$ of $\mathbb{Z}_{F}$ divisible by all $\mathfrak{p} \in \mathcal{P}$, the group $\mathcal{O}_{0}(\mathfrak{N})^{1} /\{ \pm 1\}$ is torsion-free.

Proof. If $q \in \mathcal{S}$ is odd, then $q-1=\left[\mathbb{Q}\left(\zeta_{2 q}\right): \mathbb{Q}\right] \leqslant\left[F\left(\zeta_{2 q}\right): \mathbb{Q}\right]=2[F: \mathbb{Q}]$, so that $q \leqslant 2[F: \mathbb{Q}]+1$. This proves $(1)$.

To prove (2), assume for a contradiction that there exists an element $x \in$ $\mathcal{O}_{0}(\mathfrak{N})^{1}$ such that $x$ has prime order $q$ modulo $\{ \pm 1\}$. Replacing $x$ by $-x$ if necessary, we may assume that $x$ has order $2 q$ : if $q$ is odd then either $x$ 
or $-x$ has order $2 q$; if $q=2$, then the only elements of order 2 are \pm 1 , since $B$ is a division algebra, so that $x$ has order 4 . In particular, $F(x)$ is a subfield of $B$ isomorphic to $F\left(\zeta_{2 q}\right)$. We claim that this is a quadratic extension of $F$, i.e. that $q$ belongs to $\mathcal{S}$ : if $F\left(\zeta_{2 q}\right)$ is not quadratic, then $x \in F$, but $x^{2}=\operatorname{nrd}(x)=1$, and $x$ does not have order $2 q$.

Let $P$ be the minimal polynomial of $x$, and let $\mathfrak{p} \in \mathcal{P}$ be the corresponding prime ideal. By definition, the image of $x$ in $\mathcal{M}_{2}\left(\mathbb{Z}_{F} / \mathfrak{N}\right)$ upper-triangular. Since $\mathfrak{p} \mid \mathfrak{N}$, this implies that $P$ is not irreducible modulo $\mathfrak{p}$ : a contradiction.

In our search, we extract ATR number fields of degree up to 6 and discriminants with absolute value up to $10^{6}$ from the PARI number fields database [25, 20]. Then, using the algorithms from [38] implemented in the computer algebra system Magma [7], we compute 30,000 Kleinian quaternion algebras $B$ whose maximal orders $\mathcal{O}_{\max } \subset B$ are such that $\Gamma\left(\mathcal{O}_{\max }\right)$ has covolume at most 40. Using the sufficient condition of Lemma 5.2, we find many orders $\mathcal{O}_{0}(\mathfrak{N})$ such that $\Gamma=\Gamma\left(\mathcal{O}_{0}(\mathfrak{N})\right)$ is torsion-free, and therefore acts freely on $\mathfrak{H}^{3}$, and hence we produce many arithmetic hyperbolic 3-manifolds $Y=\mathfrak{H}^{3} / \Gamma$.

Remark 5.3. The usual way of proving that arithmetic groups have a torsion-free subgroup of finite index is to consider principal congruence subgroups, which have large index. Lemma 5.2 allows us to use groups of the form $\Gamma\left(\mathcal{O}_{0}(\mathfrak{N})\right)$, which have smaller covolume, reducing the cost of the computation.

5.2. The choice of the covering. Theorem 3.5 implies that in order to produce examples as in Proposition 1.5 using Sunada's method, we need to look for $G$-coverings $X \rightarrow Y$, where $G$ is a finite group that admits a $G$-relation $\Theta=U_{1}-U_{2}$ that is not a $\mathbb{Z}_{(p)}[G]$-relation. For $p=2$, we take $G$ and $\Theta$ as in Example 2.5, and for $p$ odd, we use the relation of Example 2.6.

In order to find many such $G$-coverings of every manifold $Y=\mathfrak{H}^{3} / \Gamma$ obtained in Section 5.1, we first compute a finite presentation of $\Gamma$, using the algorithms of [24]. Since $\Gamma \cong \pi_{1}(Y), G$-coverings of $Y$ correspond to surjective homomorphisms $\Gamma \rightarrow G$. Using the presentation of $\Gamma$, we enumerate surjective homomorphisms $\Gamma \rightarrow G$ up to conjugacy, using the methods described in [17, §9.1]. The complexity of the enumeration depends heavily on $\# G$, so we use the following improvement.

Let $F_{n}$ be the free group on $n$ generators. Recall that for any group $Z$ there is a canonical bijection $\operatorname{Hom}\left(F_{n}, Z\right) \cong Z^{n}$. Moreover, if $Z$ is an abelian group, then this bijection is an isomorphism of abelian groups. We will tacitly use this identification in the next result.

Proposition 5.4. Let $\Gamma$ be a group with a finite presentation

$$
1 \rightarrow R \rightarrow F_{n} \rightarrow \Gamma \rightarrow 1,
$$

where $R$ is the normal closure of the subgroup of $F_{n}$ generated by $r$ elements $w_{1}, \ldots, w_{r}$. Let $G$ be a group, let $Z$ be a subgroup of the center of $G$, and set $\bar{G}=G / Z$. Let $\bar{h}: \Gamma \rightarrow \bar{G}$ be a homomorphism, and let $\tilde{h}: F_{n} \rightarrow G$ be an arbitrary lift of $\bar{h}: F_{n} \rightarrow \bar{G}$. Let $x=\left(\tilde{h}\left(w_{i}\right)^{-1}\right)_{i=1}^{r} \in G^{r}$, and let $\phi: Z^{n} \rightarrow$ $Z^{r}$ denote the linear map induced by evaluation on the $\left(w_{i}\right)_{i=1}^{r}$. Then: 
(1) we have $x \in Z^{r}$; and

(2) the lifts $h: \Gamma \rightarrow G$ of $\bar{h}$ are exactly the homomorphisms $\tilde{h} \cdot f$, where $f$ ranges over $\phi^{-1}(x)$.

Proof. (1) Since $\tilde{h}$ is a lift of $\bar{h}: \Gamma \rightarrow \bar{G}$, we have $\tilde{h}(R) \subset Z$, and $x \in Z^{r}$.

(2) Since $Z$ is central, every lift of $\bar{h}: F_{n} \rightarrow \bar{G}$ is of the form $\tilde{h} \cdot f$, where $f \in$ $\operatorname{Hom}\left(F_{n}, Z\right)=Z^{n}$. Such a lift descends to a homomorphism $\Gamma \rightarrow G$ if and only if it vanishes on the generators of $R$. For every such generator $w$, we have $(\tilde{h} \cdot f)(w)=1$ if and only if $f(w)=\tilde{h}(w)^{-1}$, so that the homomorphism descends if and only if $\phi(f)=x$.

Applying this proposition to $G=\mathrm{GL}_{2}\left(\mathbb{F}_{p}\right), \bar{G}=\mathrm{PGL}_{2}\left(\mathbb{F}_{p}\right)$ and $Z=\mathbb{F}_{p}^{\times}$, we reduce the enumeration of $\operatorname{Hom}(\Gamma, G)$ to:

- enumerating $\operatorname{Hom}(\Gamma, \bar{G})$,

- computing inverse images under linear maps $(\mathbb{Z} / N \mathbb{Z})^{n} \rightarrow(\mathbb{Z} / N \mathbb{Z})^{r}$ with $N=p-1$, which can be done efficiently by linear algebra.

Since the enumeration algorithm is expensive, another simple improvement was useful: if there exists a surjection $\Gamma \rightarrow G$, then $\Gamma^{a b}$ surjects onto $G^{a b}$. Checking this condition before the enumeration is fast and rules out many groups.

5.3. Homology computation. Given a surjective homomorphism $\Gamma \rightarrow G$, we can compute the homology of the isospectral manifolds as follows. For every $U \leq G$, we have

$$
H_{1}(X / U, \mathbb{Z}) \cong H_{1}(Y, \mathbb{Z}[G / U]) \cong H_{1}(\Gamma, \mathbb{Z}[G / U]),
$$

and we can compute the first homology group by linear algebra from a presentation of the group $\Gamma$. Recall that we want to find examples of $G$ coverings $X \rightarrow Y$ such that $\# H_{1}(X / \Theta, \mathbb{Z})\left[p^{\infty}\right] \neq 1$.

Since linear algebra over $\mathbb{Z}$ is costly, we used the following strategy: we first compute $H_{1}\left(X / U_{j}, \mathbb{Z}\right) \otimes_{\mathbb{Z}} \mathbb{F}_{p} \cong H_{1}\left(X / U_{j}, \mathbb{F}_{p}\right)$ for $j=1$, 2, which only uses linear algebra over $\mathbb{F}_{p}$, and we only compute the homology over $\mathbb{Z}$ if $\operatorname{dim}_{\mathbb{F}_{p}} H_{1}\left(X / \Theta, \mathbb{F}_{p}\right) \neq 0$. That condition by itself already implies that $H_{1}\left(X / U_{1}, \mathbb{Z}\right)\left[p^{\infty}\right] \not H_{1}\left(X / U_{2}, \mathbb{Z}\right)\left[p^{\infty}\right]$. In practice, when $p \geq 5$, this condition often yields examples in which $H_{1}\left(X / U_{j}, \mathbb{Z}\right)\left[p^{\infty}\right]$ is actually trivial for one $j$, and isomorphic to $\mathbb{Z} / p \mathbb{Z}$ for the other one.

By computing explicit examples, we obtain Proposition 1.5, which we restate here for convenience.

Proposition 5.5. Let $p \leq 71$ be a prime number. Then there exist strongly isospectral closed hyperbolic 3-manifolds $M_{1}$ and $M_{2}$ such that

$$
\# H_{1}\left(M_{1}, \mathbb{Z}\right)\left[p^{\infty}\right] \neq \# H_{1}\left(M_{2}, \mathbb{Z}\right)\left[p^{\infty}\right] .
$$

Moreover, if $2<p \leq 71$, then there exist 3 -manifolds $M_{1}$ and $M_{2}$ as above with

$$
\# H_{1}\left(M_{1}, \mathbb{Z}\right)\left[p^{\infty}\right]=1, \quad \text { and } \quad \# H_{1}\left(M_{2}, \mathbb{Z}\right)\left[p^{\infty}\right]=p
$$




\section{EXAMPLES}

In this section, we give several numerical examples, illustrating some features of the connection between the torsion on the homology of isospectral manifolds, and the $G$-module structure of the integral homology of their covering manifold.

Example 6.1. Let $G=\mathrm{GL}_{2}\left(\mathbb{F}_{37}\right)$, let $U_{1}, U_{2}$ be as in Example 2.6. Using the notation of Section 5.1, let $F$ be the ATR quartic number field generated by a root $t$ of $x^{4}-2 x-1$. Consider the Kleinian quaternion algebra

$$
B=\left\langle 1, i, j, k \mid i^{2}=j^{2}=k^{2}=i j k=-1\right\rangle_{F}
$$

over $F$. Let $\mathfrak{N}$ be the principal ideal of $\mathbb{Z}_{F}$ generated by $t^{2}-t-2$, which has norm 22 , and let $\Gamma_{1}=\Gamma\left(\mathcal{O}_{0}(\mathfrak{N})\right)$, which is well-defined up to conjugacy in $\mathrm{PSL}_{2}(\mathbb{C})$. A fundamental domain for $\Gamma_{1}$ acting on the ball model of $\mathfrak{H}^{3}$ is displayed in Figure 1. The group $\Gamma_{1}$ has a presentation by generators and relations as follows:

$$
\begin{aligned}
\Gamma_{1} \cong\langle a, b, c, d \quad| & b^{-1} d^{-1} c d^{2} c d^{-1} c b^{-1} c^{-1}=1, \\
& a b c^{-1} b^{-1} c^{-1} a b a^{-2} b=1, \\
& d^{-2} c b^{-1} a^{-2} b a^{-2} d^{-2} c^{-1}=1, \\
& c^{-1} a b a^{-2} d^{-1} c d^{2} a^{3} b c^{-2} d=1, \\
& \left.b^{-1} a^{-1} b^{-1} d^{-1} c d^{2} a b a^{-2} d^{-3} c b^{-1}=1\right\rangle .
\end{aligned}
$$

Let $Y=\mathfrak{H}^{3} / \Gamma_{1}$. There is a surjective group homomorphism $\Gamma_{1} \rightarrow \mathrm{GL}_{2}\left(\mathbb{F}_{37}\right)$, given by

$$
a \mapsto\left(\begin{array}{cc}
11 & 1 \\
28 & 26
\end{array}\right), \quad b \mapsto\left(\begin{array}{cc}
5 & 7 \\
30 & 32
\end{array}\right), \quad c \mapsto\left(\begin{array}{cc}
8 & 20 \\
35 & 29
\end{array}\right), \quad d \mapsto\left(\begin{array}{cc}
20 & 6 \\
26 & 17
\end{array}\right),
$$

which gives rise to a $G$-covering $X \rightarrow Y$ of arithmetic hyperbolic 3-manifolds. A direct computation, as described in Section 5.3 , yields that

$$
\begin{aligned}
& H_{1}\left(X / U_{1}, \mathbb{Z}\right) \cong \mathbb{Z}^{14} \times C_{2}^{2} \times C_{4}^{12} \times C_{8} \times C_{3} \times C_{9}^{2} \times C_{11} \times C_{1151} \times C_{11317}, \\
& H_{1}\left(X / U_{2}, \mathbb{Z}\right) \cong H_{1}\left(X / U_{1}, \mathbb{Z}\right) \times C_{37},
\end{aligned}
$$

where $C_{n}$ denotes the cyclic group of order $n$. Here, we have $\operatorname{vol}\left(X / U_{1}\right)=$ $\operatorname{vol}\left(X / U_{2}\right) \approx 1106.067$.

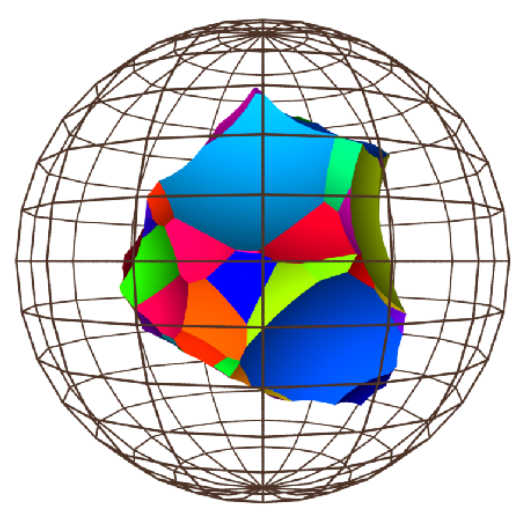

Figure 1. A fundamental domain of the Kleinian group $\Gamma_{1}$ 
It follows from Corollary 4.4 , that the multiplicity of $I=\rho(\chi, \mathbf{1})$ (see Section 4 for the definition) in the $G$-module $H_{1}(X, \mathbb{Q})$ is odd. In this particular case, we computed directly that this multiplicity is 3 .

Example 6.2. The following example shows that the $p$-torsion subgroups of two isospectral manifolds may have the same order, but be non-isomorphic.

Let $G=\mathrm{GL}_{2}\left(\mathbb{F}_{19}\right)$. Let $F$ be the ATR number field generated by a root $t$ of $x^{4}-x^{3}-3 x-1$, and let $B$ be the Kleinian quaternion algebra

$$
B=\left\langle 1, i, j, k \mid i^{2}=j^{2}=k^{2}=i j k=-1\right\rangle_{F} .
$$

Let $\mathfrak{N}$ be the principal ideal of $\mathbb{Z}_{F}$ generated by $-t^{3}+t^{2}+2 t+2$, of norm 44 , and let $\Gamma_{2}=\Gamma\left(\mathcal{O}_{0}(\mathfrak{N})\right)$, as in Section 5.1. As in the previous example, $\Gamma_{2}$ is well-defined up to conjugacy in $\mathrm{PSL}_{2}(\mathbb{C})$. A fundamental domain for $\Gamma_{2}$ is displayed in Figure 2. The hyperbolic manifold $Y=\mathfrak{H}^{3} / \Gamma_{2}$ has a $G$-covering $X \rightarrow Y$ (which we do not give explicitly this time), such that

$$
H_{1}\left(X / U_{1}, \mathbb{Z}\right)\left[19^{\infty}\right] \cong C_{19} \times C_{19}, \quad H_{1}\left(X / U_{2}, \mathbb{Z}\right)\left[19^{\infty}\right] \cong C_{19^{2}} .
$$

Here, $\operatorname{vol}\left(X / U_{1}\right)=\operatorname{vol}\left(X / U_{2}\right) \approx 985.386$.

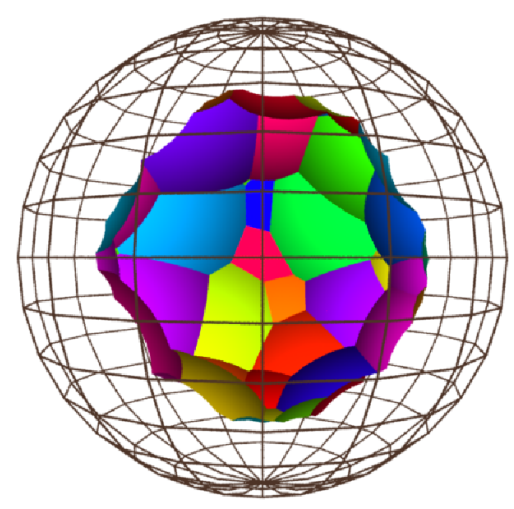

Figure 2. A fundamental domain of the Kleinian group $\Gamma_{2}$

Corollary 4.4 implies that the multiplicity of $I$ in the $G$-module $H_{1}(X, \mathbb{Q})$ is even, and in fact, in this example it is 0. Moreover, unlike in the previous example, the non-isomorphic torsion cannot be detected by the regulator constant of the $G$-module $H_{1}(X, \mathbb{Z})$.

Example 6.3. In the final example, we exhibit isospectral manifolds whose size of torsion homology differs by a square. Such an example has interesting representation theoretic features, as we will explain below.

Let $G=\mathrm{GL}_{2}\left(\mathbb{F}_{5}\right)$, and let $\Theta=U_{1}-U_{2}$ be as in Example 2.6. Let $F$ be the ATR number field generated by a root $t$ of $x^{4}-x^{3}-2 x-1$, and let $B$ be the Kleinian quaternion algebra

$$
B=\left\langle 1, i, j, k \mid i^{2}=j^{2}=k^{2}=i j k=-1\right\rangle_{F} .
$$

Let $\mathfrak{N}$ be the principal ideal of $\mathbb{Z}_{F}$ generated by $-t^{2}+2$, of norm 23 , and let $\Gamma_{3}=\Gamma\left(\mathcal{O}_{0}(\mathfrak{N})\right)$, as in Section 5.1. As in the previous examples, $\Gamma_{3}$ is well-defined up to conjugacy in $\mathrm{PSL}_{2}(\mathbb{C})$. A fundamental domain for $\Gamma_{3}$ 


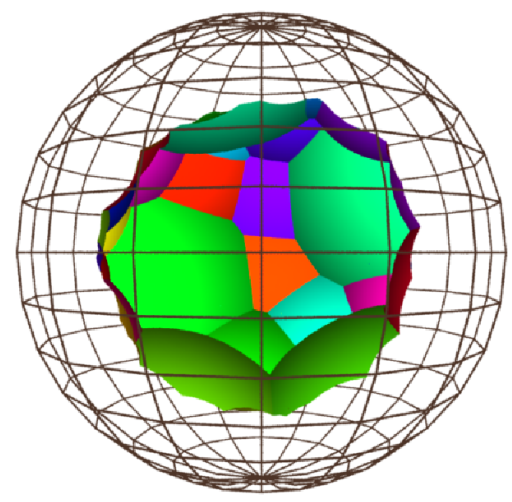

Figure 3. A fundamental domain of the Kleinian group $\Gamma_{3}$

is displayed in Figure 3. The hyperbolic manifold $Y=\mathfrak{H}^{3} / \Gamma_{3}$ has a $G$ covering $X \rightarrow Y$ such that

$$
H_{1}\left(X / U_{1}, \mathbb{Z}\right)\left[5^{\infty}\right] \cong C_{5}^{3}, \quad H_{1}\left(X / U_{2}, \mathbb{Z}\right)\left[5^{\infty}\right] \cong C_{5} .
$$

Here, $\operatorname{vol}\left(X / U_{1}\right)=\operatorname{vol}\left(X / U_{2}\right) \approx 223.790$.

Since the quotient $\# H_{1}\left(X / U_{1}, \mathbb{Z}\right)_{\text {tors }} / \# H_{2}\left(X / U_{1}, \mathbb{Z}\right)_{\text {tors }}$ is a square, Corollary 3.12 together with equation 1.2 imply that the regulator constants $\mathcal{C}_{\Theta}\left(H_{2}(X, \mathbb{Q})\right) \equiv \mathcal{C}_{\Theta}\left(H_{1}(X, \mathbb{Q})\right)$ are trivial modulo squares, which we confirm by computing that the multiplicity of $I$ in $H_{1}(X, \mathbb{Q})$ is 2 . In particular, the non-triviality of $\# H_{1}(X / \Theta)$ tors cannot be detected in the rational homology of $X$. However, by Corollary 3.13 , the non-triviality of $\# H_{1}(X / \Theta, \mathbb{Z})_{\text {tors }}$ is detected in the $G$-module structure of the integral homology via the equality $\operatorname{ord}_{5}\left(\mathcal{C}_{\Theta}\left(H_{2}(X, \mathbb{Z})\right)\right)=2$.

We can obtain similar examples of interplay between the homological torsion and representation theory for $p=2$ using Proposition 4.6 .

\section{REFERENCES}

[1] A. Bartel, On Brauer-Kuroda type relations of $S$-class numbers in dihedral extensions, J. Reine Angew. Math. 668 (2012), 211-244.

[2] A. Bartel and A. Page, Group representations in the homology of 3-manifolds, arXiv:1605.04866 [math.GT] (2016).

[3] D. J. Benson, Representations and Cohomology, Vol. I, Cambridge Studies in Advanced Mathematics 30, Cambridge University Press (1995).

[4] N. Bergeron and A. Venkatesh, The asymptotic growth of torsion homology for arithmetic groups, J. Inst. Math. Jussieu no. 2 (2013), 391-447.

[5] N. Bergeron, M. H. Şengün and A. Venkatesh, Torsion homology growth and cycle complexity of arithmetic manifolds, Duke Math. J. 165 no. 9 (2016), 1629-1693.

[6] R. Boltje, Class group relations from Burnside ring idempotents, J. Number Theory 66 (1997), 291-305.

[7] W. Bosma, J. Cannon and C. Playoust. The Magma algebra system. I. The user language. J. Symbolic Comput., 24 (1997) (3-4):235-265.

[8] J. F. Brock and N. M. Dunfield, Injectivity radii of hyperbolic integer homology 3-spheres, Geom. Topol. 19 no. 1 (2015), 497-523.

[9] K. S. Brown, Cohomology of Groups, GMT 87, Springer (1982).

[10] F. Calegari and A. Venkatesh, A torsion Jacquet-Langlands correspondence, arXiv:1212.3847v1 [math.NT] (2012). 
[11] J. Cheeger, Analytic torsion and the heat equation, Ann. of Math. 109 (1979), 259322.

[12] C. W. Curtis and I. Reiner, Methods of Representation Theory, with Applications to Finite Groups and Orders, Vol. 2, John Wiley and Sons (1987).

[13] T. Dokchitser and V. Dokchitser, Regulator constants and the parity conjecture, Invent. Math. 178 no. 1 (2009), 23-71.

[14] C. Gordon, Survey of isospectral manifolds. Handbook of differential geometry, Vol. I (2000), 747-778.

[15] C. Gordon, P. Perry and D. Schueth, Isospectral and isoscattering manifolds: a survey of techniques and examples, Geometry, spectral theory, groups, and dynamics, Contemp. Math. 387, Amer. Math. Soc. (2005), 157-179.

[16] A. Hatcher, Algebraic Topology, Cambridge University Press (2002).

[17] D. F. Holt, B. Eick, and E. A. O'Brien, Handbook of Computational Group Theory, Discrete Mathematics and its Applications (Boca Raton). Chapman \& Hall (2005).

[18] A. Ikeda, On the spectrum of a Riemannian manifold of positive constant curvature, Osaka J. Math. 17 no. 1 (1980), 75-93.

[19] M. Kac, Can one hear the shape of a drum? Amer. Math. Monthly 73 (1966), 1-23.

[20] The LMFDB Collaboration, The L-functions and Modular Forms Database (2015), http://www.lmfdb.org

[21] C. Maclachlan and A. W. Reid, The Arithmetic of Hyperbolic 3-Manifolds, Graduate Texts in Mathematics 219, Springer (2003).

[22] W. Müller, Analytic torsion and R-torsion of Riemannian manifolds, Adv. Math. 28 (1978), 233-305.

[23] W. Müller, Analytic torsion and R-torsion for unimodular representations, J. Amer. Math. Soc. 6 (1993), 721-753.

[24] A. Page, Computing arithmetic Kleinian groups, Math. Comp. 84 no. 295 (2015) 2361-2390.

[25] The PARI Group, PARI/GP version 2.7.5, Bordeaux (2015), http://pari.math. u-bordeaux.fr/

[26] G. Perelman, The entropy formula for the Ricci flow and its geometric applications, arXiv:math.DG/0211159 (2002).

[27] G. Perelman, Ricci flow with surgery on three-manifolds. arXiv:math.DG/0303109 (2003).

[28] G. Perelman, Finite extinction time for the solutions to the Ricci flow on certain three-manifolds, arXiv:math.DG/0307245 (2003).

[29] I. Piatetski-Shapiro, Complex Representations of $\mathrm{GL}(2, K)$ for Finite Fields $K$, Contemporary Mathematics 16, AMS (1983).

[30] D. B. Ray and I. M. Singer, $R$-torsion and the Laplacian on Riemannian manifolds, Advances in Math. 7 (1971), 145-210.

[31] I. Reiner, Maximal Orders, Academic Press (1975).

[32] S. Rosenberg, The Laplacian on a Riemannian Manifold, LMSST 31, Cambridge University Press (1997).

[33] I. R. Shafarevich, Basic Algebraic Geometry, Springer (1974).

[34] B. de Smit, Generating arithmetically equivalent number fields with elliptic curves in: J. P. Buhler (Ed.), Algorithmic Number Theory, Lecture Notes in Computer Science 1423, Springer (1998) 392-399.

[35] T. Sunada, Riemannian coverings and isospectral manifolds, Annals Math. 121 no. 1 (1985), 169-186.

[36] J. Tate, Les conjectures de Stark sur les fonctions $L$ d'Artin en $s=0$, Progress in Mathematics 47, Birkhuser (1984).

[37] M.-F. Vignéras, Variétés riemanniennes isospectrales et non isomtriques, Ann. of Math. (2) 112 no.1 (1980), 21-32.

[38] J. Voight, Identifying the matrix ring: algorithms for quaternion algebras and quadratic forms. Quadratic and higher degree forms, Dev. Math. 31, Springer (2013), 255-298. 